\title{
COHOMOLOGICAL PERIODICITY IN GRAPH PRODUCTS OF COMBINATORIALLY ASPHERICAL GROUPS
}

\author{
by K. J. HORADAM
}

(Received 28 October, 1991)

1. Introduction. The notion of a group $G$ having periodic cohomology after $k$ steps was introduced by Talelli in [10], and is equivalent to having the functors $H^{m}(G,-)$ and $H^{m+q}(G,-)$ naturally isomorphic for some $q \geq 1$ and all $m \geq k+1$. It extends to infinite groups the long-understood phenomenon of cohomological periodicity for finite groups (for which $k=0$ ).

The nature of the torsion elements in a group seems to control the periodicity of its cohomology, and in [11], Talelli suggests that any group with periodic cohomology after 1 step arises as the fundamental group of a graph of finite groups with periodic cohomology.

It is natural to enquire when periodicity is inherited by fundamental groups of graphs of groups from other families whose cohomology is periodic after $k$ steps. Combinatorically aspherical (CA) groups have a particularly simple cohomological description, and have cohomological period 2 after 2 steps, characterised by cup product action [5]. Their torsion elements are precisely the conjugates of powers of roots of their relators. Examples are one-relator groups, small cancellation groups and knot groups. In [7] the author analysed the cohomology ring structure of an HNN-extension of a CA group by determining a condition sufficient to ensure that the extension has periodic cohomology, characterized by cup product action. The necessity of this condition was not investigated.

In this paper, a sufficiency result is proved for any fundamental group of a graph of CA groups: if the fundamental group (termed a "graph product" here) has finite "uniform order" then it has periodic cohomology, and a cup product action completes a chain isomorphism on the Mayer-Vietoris sequence of the graph product. If all edge groups (or all vertex groups) are finitely related and the graph is finite, the graph product has finite uniform order. Consequently, when all the edge and vertex groups are finitely presented and the graph is finite, the cohomology ring of a graph product with trivial coefficients in a principal ideal domain is finitely presented, and a presentation can be found.

Furthermore, the necessity of the uniform order condition is proved: a periodic graph product must have finite uniform order. Proofs use the exact form of the cup product, calculated in [6], for the graph product. As an intermediate step, the concept of "structural period" is introduced, and related to the usual period.

General references are [2] for group cohomology and [3] for CA groups.

I wish to thank Olympia Talelli for first interesting me in inheritance of periodic properties and for her valuable comments on an earlier draft of this paper. A generalized version of some of the results in [11] is presented in Section 2. I would also like to thank David Rusin for prompting the study of necessity of uniform order.

2. Periodicity in graph products. Let $D=(V, E)$ be a connected directed graph, and let $(\mathscr{G}, D)$ be a graph of groups [2, p. 179], so that for each vertex $v \in V$ there is a presentation $G_{v}=\left\langle X_{v} \mid R_{v}\right\rangle$ and for each edge $e \in E$ there is a presentation of a subgroup 
$K_{e}=\left\langle Y_{e} \mid S_{e}\right\rangle$ of $G_{s e}$ and a group monomorphism $\phi_{e}: K_{e} \rightarrow G_{t e}$, where se and te are the source and terminus vertices of $e$, respectively. Let $H$ be the graph product (fundamental group) of $(G, D)$; that is, for a choice of maximal tree $T$ in $D, H$ has the presentation

$$
H=\left\langle X_{v}, v \in V ; e, e \in E \mid R_{v}, v \in V ; e=1, e \in T ; e \phi_{e}(y)=y e, y \in Y_{e}, e \in E\right\rangle .
$$

If $H$ has period $q$ after $k$ steps [10, p. 179], so do all its subgroups [10, 1.3], so in particular, all the vertex groups $G_{v}$ and all the edge groups $K_{e}$ have period $q$ after $k$ steps. It is natural to ask when a graph product $H$ inherits cohomological periodicity from its vertex groups, and, if it does, whether this periodicity may be characterised by cup product action. These questions have been considered by Bieri and Talelli $[1,11]$ and the author [7]. It is known that periodicity is not always inherited $([7,3.7],[11,2.2 . b])$.

When a minimum cohomological period is characterised by cup product action we borrow the terminology from periodic finite groups and term a characterising class a maximal generator.

Definition 2.1 (i) If a group $G$ has (minimum) period $q$ after $k$ steps a (maximal) generator of degree $q$ for $G$ is a $g \in H^{q}(G ; \mathbb{Z})$ such that for any $G$-module $A$,

$$
g \cup-: H^{m}(G ; A) \rightarrow H^{m+q}(G ; A)
$$

is an isomorphism for $m>k$ and an epimorphism for $m=k$.

(ii) If a graph product $H$ has a generator in $H^{q}(H ; \mathbb{Z})$ then $q$ is termed a structural period of $H$.

A structural period is so called because the degree $q$ generator will complete a chain isomorphism on the Mayer-Vietoris sequence of $H$. Clearly the minimum structural period, when it exists, will be a multiple of the minimum period of $H$. 179]) is:

For any $\mathbb{Z} H$-module $A$ the Mayer-Vietoris exact cohomology sequence (cf. [2, p.

$$
\ldots \rightarrow H^{m}(H ; A) \stackrel{i^{*}}{\longrightarrow} \prod_{v \in V} H^{m}\left(G_{v} ; A\right) \stackrel{C^{*}}{\longrightarrow} \prod_{e \in E} H^{m}\left(K_{e} ; A\right) \stackrel{\pi^{*}}{\longrightarrow} H^{m+1}(H ; A) \rightarrow \ldots,
$$

where $i^{*}=\prod_{v \in V} r e s_{G_{u}}^{*}, C^{*}$ is determined from the edge monomorphisms to be

$$
C^{*}\left(\prod_{v \in V} f_{v}\right)=\prod_{e \in E}\left(c_{e}^{*} \operatorname{res}_{\phi_{e}\left(K_{e}\right)}^{*}\left(f_{t e}\right)-\operatorname{res}_{K_{e}}^{*}\left(f_{s e}\right)\right), \quad f_{v} \in H^{q}\left(G_{v} ; \mathbb{Z}\right),
$$

and the isomorphism $c_{e}^{*}$ is induced from conjugation by $e$ in $H$. A family $\left\{f_{v} \in\right.$ $\left.H^{q}\left(G_{v} ; \mathbb{Z}\right), v \in V\right\}$ of degree $q$ generators is called compatible if

$$
c_{e}^{*} r e s_{\phi_{e}\left(K_{e}\right)}^{*}\left(f_{r e}\right)=r e s_{K_{e}}^{*}\left(f_{s e}\right), \quad e \in E .
$$

In [11, 2.1] Talelli shows that a graph product of finite groups has period $q$ after 1 step if and only if every vertex group $G_{v}$ has period $q$ and there is a compatible family of maximal generators. Her proof is, in fact, in terms of structural period (periods of such graph products are always structural), and her argument adapts to any graph product whose vertex groups have periodic generators of bounded degree.

THEOREM 2.2 [Talelli]. The graph product $H$ has structural period $q$ after some steps if and only if every vertex group $G_{v}$ has period $q$ after some steps and there is a compatible family $\left\{f_{v}, v \in V\right\}$ of generators of degree $q$. 
Proof. If every vertex group $G_{v}$ has period $q$ after $k-1$ steps and $\left\{f_{v} \epsilon\right.$ $\left.H^{a}\left(G_{v} ; \mathbb{Z}\right), v \in V\right\}$ is a compatible family then $C^{*}\left(\prod_{v \in V} f_{v}\right)=0$. Since (1) is exact, there is an element $f \in H^{q}(H ; \mathbb{Z})$ with $\operatorname{res}_{G_{v}}^{*}(f)=f_{v}, v \in V$. Shapiro's Lemma implies that $f_{e}=\operatorname{res}_{K_{e}}^{*}\left(f_{s e}\right)=\operatorname{res}_{K_{e}}^{*}(f)$ is a degree $q$ generator for $K_{e}, e \in E$. By naturality of cup product there is a skew-commutative diagram

$$
\begin{aligned}
& \prod_{e \in E} H^{m-1}\left(K_{e} ; A\right) \longrightarrow H^{m}(H ; A) \longrightarrow \prod_{v \in V} H^{m}\left(G_{v} ; A\right) \longrightarrow \prod_{e \in E} H^{m}\left(K_{e} ; A\right) \\
& \prod_{e \in E} f_{e} \cup-\downarrow \quad f \cup-\downarrow \quad \prod_{U \in \mathcal{V}} f_{v} \cup-\downarrow \quad \prod_{e \in E} f_{e} \cup-\downarrow \\
& \prod_{e \in E} H^{m-1+q}\left(K_{e} ; A\right) \longrightarrow H^{m+q}(H ; A) \rightarrow \prod_{v \in V} H^{m+q}\left(G_{v} ; A\right) \rightarrow \prod_{e \in E} H^{m+q}\left(K_{e} ; A\right)
\end{aligned}
$$

and the 5-Lemma implies $H$ has structural period $q$ after $k$ steps. Conversely, if $f$ is a degree $q$ generator for $H$, then by Shapiro's Lemma, $\operatorname{res}_{G_{v}}^{*}(f)=f_{v} \in H^{q}\left(G_{v} ; \mathbb{Z}\right)$ is a degree $q$ generator for $G_{v}, v \in V$. From (1) it follows that $C^{*}\left(\prod_{v \in V} f_{v}\right)=0$, so the family $\left\{f_{v}, v \in V\right\}$ is compatible.

A periodic graph product will be structurally periodic when additional conditions apply (as they do in [11]).

THEOREM 2.3 [Talelli]. If $H$ has period $q$ after $k \geq 1$ steps and if $H^{m}\left(K_{e} ; P\right)=$ $H^{m}\left(G_{v} ; P\right)=0$ for all $q \leq m \leq q+k, e \in E, v \in V$ and projective $\mathbb{Z} H$-modules $P$, then $H$ has structural period $q$.

Proof. By (1), $H^{m}(H ; P)=0$ for all $q+1 \leq m \leq q+k$ and all $P$. Hence by $[11,1.1]$ there is a generator of degree $q$ for $H$.

We remark that Theorem 2.1 of [11] illustrates the application of (2.2) and (2.3) above for the case $k=1$ while the results below illustrate the case $k=3$. A natural query is: are there examples for every $k \geq 1$ ?

3. Graph products of $\mathbf{C A}$ groups. Every subgroup of a $\mathrm{CA}$ group has a $\mathrm{CA}$ presentation $[3,2.5]$. We assume from now on that $G_{v}, v \in V$ and $K_{e}, e \in E$ are concise presentations of CA groups. If the edge groups $K_{e}$ are all trivial, the graph product is CA $[3,4.6]$, and its periodic behaviour is known. If the edge groups are all torsionfree, $H$ is cohomologically indistinguishable from a CA group after dimension 3 .

Assume for the moment that at least one nontrivial edge group has torsion.

The main periodicity results in the torsion case depend on the fact that the torsion elements in a CA group are precisely the conjugates of powers of roots of the relators. Notation follows $[6,7]$. For each $v \in V$, let $F_{v}$ be the free group on $X_{v}$, and for each $e \in E$ write each relator $s$ of $S_{e}$ as a power of its root: $s=t_{s}^{n_{s}} \in S_{e}$, for $n_{s}$ maximal. By the torsion condition [8, Thm. 3], there are uniquely determined relators $\rho_{s}=\zeta_{s}^{v_{s}} \in R_{s e}$ and $\rho_{s}^{\prime}=\left(\zeta_{s}^{\prime}\right)^{v_{s}^{\prime}} \in R_{t e}$ such that for suitable $y_{s} \in F_{s e}, y_{s}^{\prime} \in F_{t e}$ and for uniquely determined integers $K_{s}$ and $K_{s}^{\prime}$

$$
t_{s}=y_{s} \zeta_{s}^{\kappa_{s}} y_{s}^{-1} \in G_{s e}, \quad \phi_{e}\left(t_{s}\right)=y_{s}^{\prime}\left(\zeta_{s}^{\prime}\right)^{\kappa_{s}^{\prime}}\left(y_{s}^{\prime}\right)^{-1} \in G_{t e}
$$


Write $k_{s}=\kappa_{s} /\left(\kappa_{s}, v_{s}\right)$ and $k_{s}^{\prime}=\kappa_{s}^{\prime} /\left(\kappa_{s}^{\prime}, v_{s}^{\prime}\right)$. (Note $n_{s}=v_{s} /\left(\kappa_{s}, v_{s}\right)=v_{s}^{\prime} /\left(\kappa_{s}^{\prime}, v_{s}^{\prime}\right)$; and if $n_{s}=1, \kappa_{s}=\kappa_{s}^{\prime}=0$.)

Thus each $s \in S_{e}, e \in E$ uniquely determines three integers: $n_{s}, k_{s}$ and $k_{s}^{\prime}$. These turn determine a crucial structure constant of $H$.

Definition 3.1. Let $S_{e}^{+}=\left\{s=t_{s}^{n_{s}} \in S_{e}, n_{s}\right.$ maximal $\left.: n_{s} \geq 2\right\}$ for each $e \in E$. Define the uniform order $\psi$ of $H$ to be the smallest positive integer, when it exists, such that

$$
\left(k_{s}\right)^{\psi}-1 \equiv\left(k_{s}^{\prime}\right)^{\psi}-1 \equiv 0 \bmod n_{s}, \quad s \in S_{e}^{+}, \quad e \in E,
$$

and define $\psi=\infty$ otherwise. If $\psi$ is finite, define $p_{s}$ and $p_{s}^{\prime}$ for $s \in S_{e}^{+}, e \in E$, by

$$
\left(k_{s}\right)^{\psi}-1=p_{s} n_{s}, \quad\left(k_{s}^{\prime}\right)^{\psi}-1=p_{s}^{\prime} n_{s}
$$

so that $\psi=1$ if and only if $k_{s}=k_{s}^{\prime}=1$ and $p_{s}=p_{s}^{\prime}=0$.

Such a $\psi$ will always exist if $\left\{n_{s}: s \in S_{e}^{+}, e \in E\right\}$ is bounded above, for example, if $D$ is finite and all the edge groups $K_{e}$ (or all the vertex groups $G_{v}$ ) are finitely related.

For each $v \in V$, let $L^{v}$ be the Lyndon resolution $[4,2.1]$ for $G_{v}$, and let $R_{v}^{+}$be the set of all relators which are proper powers, i.e. $R_{v}^{+}=\left\{r=z_{r}^{n_{r}} \in R_{v}, n_{r}\right.$ maximal: $\left.n_{r} \geq 2\right\}$. For each $e \in E$, define $L^{e}$ similarly ( $S_{e}^{+}$is already defined). The edge monomorphisms determine a $\mathbb{Z} H$-chain map

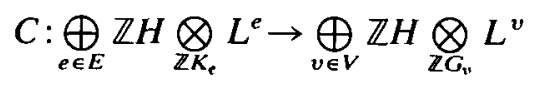

whose (unaugmented) mapping cone $M$ induces the Mayer-Vietoris sequence (1), where $\pi^{*}$ is induced from the canonical projection $\pi: M \rightarrow \underset{e \in E}{\bigoplus} \mathbb{Z} H \underset{\mathbb{Z} \mathcal{K}_{e}}{\bigotimes} L^{e}$ of degree -1 .

Lemma 3.2 [6, 3.3], cf. [7, 2.2]. In even dimensions the cohomology boundary map on $M$ is given for $f \in \operatorname{Hom}_{\mathbb{Z} H}\left(M_{*}, A\right), v \in V$ and $e \in E$ by

$$
\begin{aligned}
\delta_{0}(f)(x) & =(x-1) f(v), \quad x \in X_{v} \\
\delta_{0}(f)(e) & =e f(t e)-f(s e) \\
\delta_{2}(f)(r) & =\left(z_{r}-1\right) f(r), \quad r \in R_{v} \\
\delta_{2}(f)(e, s) & =-\sum_{y \in Y_{e}}(\partial s / \partial y) f(e, y)+e \sum_{r \in R_{t e}}\left(\partial \phi_{e}(s) / \partial r\right) f(r)-\sum_{r \in R_{s e}}(\partial s / \partial r) f(r), \quad s \in S_{e} \\
\delta_{2 m}(f)(r) & =\left(z_{r}-1\right) f(r), \quad r \in R_{v}^{+} \\
\delta_{2 m}(f)(e, s) & =-\left(\sum_{j=0}^{n_{s}-1} t_{s}^{j}\right) f(e, s)+e\left(k_{s}^{\prime}\right)^{m} y_{s}^{\prime} f\left(\rho_{s}^{\prime}\right)-\left(k_{s}\right)^{m} y_{s} f\left(\rho_{s}\right), \quad s \in S_{e}^{+}, \quad m \geq 2 .
\end{aligned}
$$

Here $\partial / \partial y, \partial / \partial r$ refers to differentiation in the free group generated by $Y_{e}, \bigcup_{v \in V}\left(R_{v} \cup X_{v}\right)$, respectively.

4. Periodicity in graph products of $\mathbf{C A}$ groups. If $H$ has periodic cohomology after some steps, any period is necessarily even since the finite cyclic subgroups of $H$ inherit the period of $H[10,1.3]$ and also have period 2. 
We show firstly that if $H$ has finite uniform order then $H$ has finite structural period. (The specific $\mathbb{Z} H$-resolution $M$ is used.) If $\psi=1, H$ necessarily has period $2 \psi=2$ since the mapping cone $M$ itself has period 2 after 3 steps. However, no maximal generator has been found in all the cases $\psi=1$. (See (5.1) below for a positive case.)

Proposition 4.1. If $H$ has finite uniform order $\psi$, define $\lambda=\max \{\psi, 2\}$. Define $\beta_{v}: L_{2}^{v} \rightarrow \mathbb{Z}$ to be $\beta_{v}(r)=1, r \in R_{v}$. Then $\left[\beta_{v}\right]^{\lambda}$ is a generator of degree $2 \lambda$ for $G_{v}$. The mapping $\beta: M_{2 \lambda} \rightarrow \mathbb{Z}$ given by $\mathbb{Z} H$-free extension of

$$
\begin{gathered}
\beta(r)=1, \quad r \in R_{v}^{+}, \quad v \in V, \\
\beta(e, s)=p_{s}^{\prime}-p_{s}, \quad s \in S_{e}^{+}, \quad e \in E,
\end{gathered}
$$

is a $2 \lambda$-cocycle satisfying $\operatorname{res}_{G_{v}}^{*}([\beta])=\left[\beta_{v}\right]^{\lambda}, v \in V$.

Proof. By $[5,2.2],\left[\beta_{v}\right]$ is a maximal generator for $G_{v}$, so $\left[\beta_{v}\right]^{\lambda}$ is a generator of degree $2 \lambda$ for $G_{v}$. By the cup product formula $[4,4.1]$ for CA groups, $\beta_{v}^{\lambda}(r)=1=\beta(r)$, $r \in R_{v}^{+}, v \in V$. From (3.2), $\delta_{2 \lambda}(\beta)(r)=0 . \beta(r)=0$ for all $r \in R_{v}^{+}, v \in V$, and for all $s \in S_{e}^{+}$, $e \in E$,

$$
\delta_{2 \lambda}(\beta)(e, s)=-n_{s} \beta(e, s)+\left(k_{s}^{\prime}\right)^{\lambda} \beta\left(\rho_{s}^{\prime}\right)-\left(k_{s}\right)^{\lambda} \beta\left(\rho_{s}\right)=-n_{s}\left(p_{s}^{\prime}-p_{s}\right)+\left(k_{s}^{\prime}\right)^{\lambda}-\left(k_{s}\right)^{\lambda}
$$

which is 0 by (3).

THEOREM 4.2. If $H$ has finite uniform order $\psi=1, H$ has period 2 after 3 steps and structural period 4 . If $H$ has finite uniform order $\psi>1, H$ has structural period $2 \psi$ after 3 steps.

Proof. By (4.1) $\left\{\left[\beta_{v}\right]^{\lambda}, v \in V\right\}$ is a compatible family of generators of degree $2 \lambda$ so that by (2.2) $H$ has structural period $2 \lambda$.

(The result can also be obtained by direct calculation on $M$, mimicking that of $[7$, $3.3,3.4,3.5]$ for the HNN-extension. In fact, if $H$ is a free product with amalgamation the result is immediate since $H$ can be embedded in an $\mathrm{HNN}$-extension $[9, \mathrm{p} .187]$ with the same uniform order.)

Corollary 4.3. If $H$ has finite uniform order and the trivial coefficient ring $A$ is a principal ideal domain, $H^{*}(H ; A)$ is generated as a ring by elements of degree at most $\lambda+2$. If $G_{v}, v \in V$ and $K_{e}, e \in E$ are all finitely presented, and $D$ is finite, $H^{*}(H ; A)$ is finitely presented as a ring over $A$.

Proof. The first result is immediate from (4.2). The second result then follows since the $H^{m}(H ; A)$ are all finitely presented. Indeed, a presentation may be found (cf. the remarks of $[7, \S 4])$.

The results above may be extended to include the torsionfree case $\left(n_{s}=1, k_{s}=k_{s}^{\prime}=\right.$ $0, s \in S_{e}^{+}, e \in E$ ) by setting the default value $\psi=1$, since if all the $K_{e}$ are torsionfree (that is, aspherical, by $[3,1.3]$ ), the mapping cone $M$ of $H$ is the same as that of the free product $\underset{v \in V}{*} G_{v}$ after dimension 3 and hence has period 2 after 3 steps $[10,1.8]$. Since the free product is CA its period 4 is structural, hence so is that of $H$.

Assume from now on that edge groups may all be torsionfree.

Secondly, any period of $H$ greater than 2 is structural.

THeOREM 4.4 [Talelli]. If $H$ has period $2 q>2$ after $k$ steps then $2 q$ is structural. 
Proof. If $P$ is a projective $\mathbb{Z} H$-module, then $H^{m}\left(K_{e}, P\right)=H^{m}\left(G_{v}, P\right)=0$ for $m \geq 3$, $e \in E$, and $v \in V$, because a CA group has the cohomology of a free product of finite cyclic groups in dimensions $\geq 3$. Then (2.3) applies.

Aperiodic graph products (with $\psi=\infty$ ) do exist $[7,3.7]$. We show lastly that a partial converse of (4.2) holds.

Lemma 4.5. Suppose $[\alpha] \in H^{2 q}(H ; \mathbb{Z})$ is a generator of degree $2 q$ for $H$, for $\alpha: M_{2 q} \rightarrow \mathbb{Z}$. Then $\alpha(r) \equiv 1 \bmod n_{r}$, for all $r \in R_{v}^{+}\left(r \in R_{v}\right.$ if $\left.q=1\right)$ and $v \in V$.

Proof. Immediate since $\operatorname{res}_{G_{v}}^{*}([\alpha])=\left[\beta_{v}\right]^{q}$.

THEOREM 4.6. If $H$ has structural period $2 q$ after 3 steps then $H$ has finite uniform order dividing $q$.

Proof. Suppose $[\alpha]$ is a generator for $H$, for $\alpha: M_{2 q} \rightarrow \mathbb{Z}$. For $j \geq 2$, let $\left[\beta_{e}\right]=$ $\operatorname{res}_{K_{e}}^{*}\left(\left[\beta_{s e}\right]\right), e \in E$ and define $\sigma: \bigoplus_{e \in E} L_{e}^{2 j-2} \rightarrow \mathbb{Z}$ by $\sigma(s)=1, s \in S_{e}^{+}, e \in E$, so that $[\sigma]=$ $\prod_{e \in E}\left[\beta_{e}\right]^{j-1} \in \prod_{e \in E} H^{2 j-2}\left(K_{e} ; \mathbb{Z}\right)$. The cup product formula [6, 5.1] for the cohomology of $H$ in dimension $2(j+q)-1$ with coefficients in $\mathbb{Z}$ gives

$$
\begin{aligned}
\left(\alpha \cup \pi^{*}(\sigma)\right)(e, s) & =\alpha(e, s) \cdot\left(k_{s}^{\prime}\right)^{j-1} \kappa_{s}^{\prime} \pi^{*}(\sigma)\left(\rho_{s}^{\prime}\right)+k_{s}^{q} \alpha\left(\rho_{s}\right) \cdot \pi^{*}(\sigma)(e, s) \\
& =k_{s}^{q} \alpha\left(\rho_{s}\right) .1, \quad s \in S_{e}^{+}, \quad e \in E,
\end{aligned}
$$

since $\pi^{*}(\sigma)(r)=0, r \in R_{v}^{+}, v \in V$. Because $[\alpha] \cup \pi^{*}([\sigma])=\pi^{*}\left(\left[\beta_{e}\right]^{q} \cup[\sigma]\right), k_{s}^{q} \alpha\left(\rho_{s}\right)=1$, $s \in S_{e}^{+}, e \in E$. By $(4.5) \alpha\left(\rho_{s}\right) \equiv 1 \bmod v_{s}$, and $n_{s} \mid v_{s}$, so $k_{s}^{q} \equiv 1 \bmod n_{s}$ for $s \in S_{e}^{+}, e \in E$. By commutativity of cup product with $[\alpha]$, the symmetric result $\left(k_{s}^{\prime}\right)^{q} \equiv 1 \bmod n_{s}$ for $s \in S_{e}^{+}$, $e \in E$, also holds. Since $q$ satisfies (2), $H$ has finite uniform order dividing $q$.

COROLlaRY 4.7. The graph product $H$ has structural period $2 . \max \{q, 2\}$ after 3 steps if and only if $H$ has finite uniform order dividing $\max \{q, 2\}$.

COROLlary 4.8. The graph product $H$ has minimum period $2 \psi>2$ after 3 steps if and only if $H$ has finite uniform order $\psi>1$.

The results above, together with the fact that if $H$ has period 2 it has period 4 which is therefore structural, prove that for graph products of CA groups,

$$
\text { finite uniform order } \Leftrightarrow \text { structurally periodic } \Leftrightarrow \text { periodic. }
$$

5. Conclusion. Inheritance of cohomological periodicity by a graph product from its CA vertex groups depends critically on the edge group presentations and monomorphisms. From (4.5), if $H$ has $\psi=1$ and minimum structural period 2, then the 2-cocycle $\alpha$ inducing the maximal generator must satisfy

$$
\alpha(r) \equiv 1 \bmod n_{r}, \quad r \in R_{v}, \quad v \in V .
$$

By (2.2) this will occur precisely when the family of maximal generators $\left\{\left[\beta_{v}\right], v \in V\right\}$ is compatible. Equivalently from (3.2), on writing $\langle u: v\rangle$ for the image of $\partial u / \partial v$ in $\mathbb{Z}$, this will occur precisely when there exist integers $\alpha(e, y), y \in Y_{e}, e \in E$ such that for each $s \in S_{e}$,

$$
\sum_{y \in Y_{e}}\langle s: y\rangle \alpha(e, y)=\sum_{r \in R_{t e}}\left\langle\phi_{e}(s): r\right\rangle \alpha(r)-\sum_{r \in R_{s e}}\langle s: r\rangle \alpha(r) .
$$


EXAMPLE 5.1. Let $D$ be a bouquet of circles, so for all $e \in E, t e=s e=0$ (say), and let $\phi_{\mathrm{e}}$ be conjugation by $g_{\mathrm{e}} \in G_{0}$ for each $e \in E$. If $H$ has uniform order $\psi=1$ then the minimum period 2 is the minimum structural period.

Proof. For all $s \in S_{e}^{+}, e \in E, \rho_{s}=\rho_{s}^{\prime}, \kappa_{s}=\kappa_{s}^{\prime}, k_{s}=k_{s}^{\prime}$ and the images in $\mathbb{Z}$ of $e \partial\left(g_{e}^{-1} s g_{e}\right) / \partial r$ and $\partial s / \partial r$ are the same. If $\psi=1$ then $p_{s}=p_{s}^{\prime}=0$ so the mapping $\beta: M_{2} \rightarrow \mathbb{Z}$ defined as in (4.1) by $\mathbb{Z} H$-free extension of

$$
\begin{aligned}
\beta(r) & =1, & r \in R_{v}, & v \in V, \\
\beta(e, y) & =0, & y \in Y_{e}, & e \in E,
\end{aligned}
$$

is a 2-cocycle, since $\sum_{y \in Y_{e}}\langle s: y\rangle \beta(e, y)=0=\sum_{r \in R_{0}}\left(\left\langle g_{e}^{-1} s g_{e}: r\right\rangle-\langle s: r\rangle\right) \beta(r)$.

It seems unlikely a compatible family will always exist.

CONJECTURE 1 . There exists a graph product of CA groups which has uniform order $\psi=1$ and minimum structural period 4 .

If $H$ has period 2 then by (4.7) it has uniform order either 1 or 2 .

CONJECTURE 2. If a graph product of CA groups has period 2 then it has uniform order $\psi=1$.

\section{REFERENCES}

1. R. Bieri and O. Talelli, Group pairs with periodic cohomology, J. Pure Appl. Algebra 64 (1990), 229-238.

2. K. S. Brown, Cohomology of groups (GTM 87, Springer, New York, 1982).

3. I. M. Chiswell, D. J. Collins and J. Huebschmann, Aspherical group presentations, Math. Z. 178 (1981), 1-36.

4. K. J. Horadam, The cup product and coproduct for a combinatorially aspherical group, $J$. Pure Appl. Algebra 33 (1984), 41-47.

5. K. J. Horadam, The cohomology ring of a combinatorially aspherical group, J. Austral. Math. Soc. (Series A) 47 (1989), 453-457.

6. K. J. Horadam, The cup product for a graph product of combinatorially aspherical groups, Comm. Algebra 18 (1990), 3245-3262.

7. K. J. Horadam, The cohomology ring of an HNN extension of combinatorially aspherical groups, J. Pure Appl. Algebra 72 (1991), 23-32.

8. J. Huebschmann, Cohomology theory of aspherical groups and of small cancellation groups, J. Pure Appl. Algebra 14 (1979), 137-143.

9. R. C. Lyndon and P. E. Schupp, Combinatorial group theory (Springer, Berlin, 1977).

10. O. Talelli, On cohomological periodicity for infinite groups, Comment. Math. Helv. 55 (1980), 178-192.

11. O. Talelli, On groups with periodic cohomology after 1-step, J. Pure Appl. Algebra 30 (1983), 85-93.

CMR GrouP, ERL, D.S.T.O. c/o DVR2, A BLOCK, NEW WING Victoria Barracks, Melbourne Australia 3004
Department of Mathematics

R.M.I.T.

G.P.O. Box 2476V, Melbourne Australia 3001 